\title{
Sistema de informação: instrumento para tomada de decisão no exercício da gerência
}

\author{
Eliane Marina Palhares Guimarães \\ Doutoranda em enfermagem fundamental. Linha de pesquisa: \\ dinâmica e organização dos serviços de saúde e de enfermagem. \\ Mestre em ciência da informação/UFMG. Professora da Escola de \\ Enfermagem da UFMG. \\ E-mail: elianemg@enf.ufmg.br
}

\section{Yolanda Dora Martinez Évora}

Doutora em enfermagem/EERP/USP. Professora livre docente da Escola de Enfermagem de Ribeirão Preto/USP.

E-mail:ydmevora@eerp.usp.br

\section{Resumo}

Este artigo tem como objetivo analisar a utilização de sistemas de informação enquanto instrumento para a tomada de decisão no exercício da gerência. Apresenta resultados da pesquisa realizada com os coordenadores e gerentes das unidades funcionais do Hospital das Clínicas da Universidade Federal de Minas Gerais. Baseada em pesquisa descritiva, a análise dos dados revela que o modelo de gerência fundamentado em unidades funcionais é um passo importante para a reorganização institucional. Demonstra que os sistemas de informação são instrumentos importantes para o exercício da gerência. Aponta subsídios para o desenvolvimento de sistemas de informação, bem como para sua automação.

\section{Palavras-chave}

Sistemas de informação; Administração hospitalar/ tendências; Tomada de decisão; Gerência.

\section{Information system: instrument for decision making in management performance}

\begin{abstract}
This article has the objetive of analysing the use of Information Systems as a tool for management decisionmaking. It presents results of a research done with coordinators and managers of Functional Units of Hospital das Clínicas from the Federal University Minas Gerais, Brazil. The data analysis, which was based on descriptive research, demonstrates that the model of management based on Functional Units is an important step towards institutional reorganization. It demonstrates that Information Systems is an important tool to decision making process. It indicates the ways and means to Information Systems development as well as its automation.
\end{abstract}

\section{Keywords}

Information Systems; Hospital administration/tendencies; Decision making; Management.

\section{INTRODUÇÃO}

As mudanças no mundo contemporâneo têm gerado transformações radicais na forma de produção e de relação entre os indivíduos. Nas últimas décadas, a revolução provocada pela informática nos ambientes empresariais e até mesmo domésticos tem feito com que grande parte das pessoas altere seus hábitos. Tomando como base os períodos históricos da evolução da sociedade, marcados inicialmente pela produção manufatureira e, posteriormente, demarcados pela produção industrial e pós-industrial, constatamos que foram fortemente influenciados pela incorporação tecnológica nos processos de produção e que causaram grandes mudanças na forma de vida das pessoas. Com o avanço tecnológico, este processo tornou-se mais complexo. As empresas transformaram-se em organizações cada vez mais complexas, hierarquizadas, especializadas e que demandavam supervisão e gerência. Por conseguinte, a preocupação passou a ser com a autoridade, responsabilidade, planejamento, controle, coordenação e relações no trabalho (Motta, 1986).

As transformações apontam para um redirecionamento dos objetivos da organização, antes voltados para o controle da produção de bens e serviços, para outra baseada na informação, na tecnologia e no consumo. Em conseqüência, a gerência praticada nas organizações se volta para a valorização da descentralização administrativa, da comunicação informal, da flexibilidade nos processos de produção, assim como para o estímulo à iniciativa e criatividade dos indivíduos e grupos.

Nos ambientes empresariais, a automação tem ocupado papel fundamental, utilizando-se de seus recursos para o tratamento da informação necessária à tomada de decisão. A maneira como a informação é obtida, organizada, gravada, recuperada e posteriormente utilizada permite ao gerente atuar com mais segurança, aumentando a possibilidade de acerto na tomada de decisão.

\section{TEORIAS DA ADMINISTRAÇÃO: EVOLUÇÃO E TENDÊNCIAS}

Desde o início do século, as empresas foram organizadas a partir dos princípios da Teoria Científica, cujo precursor, Frederick Taylor, preconizava a divisão do 
trabalho, a disciplina, a racionalização dos métodos e sistemas de trabalho e a padronização da produção. Para garantir que os padrões de produção fossem atingidos, sugeriu a seleção, o treinamento e o controle dos trabalhadores, incluindo o pagamento pela produção gerada em cada um deles, por meio de um sistema de incentivos monetários (Motta, 1986).

Corroborando esta afirmativa, Oliveira (1997) enfatiza que os antigos paradigmas tayloristas levaram a profundas distorções nas organizações, que desumanizaram as relações no trabalho e robotizaram os indivíduos, resultando em desmotivação e, conseqüentemente, em baixa produtividade. Neste contexto, os gerentes assumiam o papel de "controladores" encarregados de fiscalizar os funcionários para garantir maior produtividade. Por sua vez, os trabalhadores eram explorados, tornavam-se cada vez mais alienados e não mantinham controle sobre sua produtividade.

Em 1916, Henry Fayol, buscando a racionalização da estrutura administrativa, propõe a adoção de uma estrutura adequada e de um funcionamento compatível com essa estrutura. Fayol salienta o princípio da unidade de comando, da divisão do trabalho, da especialização e da amplitude de controle.

$\mathrm{Na}$ década de 30, a administração passou a valorizar o recurso humano, correspondendo a um deslocamento da ênfase na organização formal para a informal, entendida como o conjunto das relações sociais não previstas em regulamentos e organogramas e nas relações de trabalho, caracterizadas pela espontaneidade e pela falta de objetivo comum.

Estas premissas foram marcantes na influência das teorias da administração posteriores que passaram a preocupar-se com o lado psicossocial que envolve o processo de trabalho. A exemplo disto, na década de 40 , a Teoria Comportamentalista reforça a preocupação com as relações das pessoas no processo de trabalho nas organizações, reconhecendo a propriedade de adaptação do ser humano e enfatizando a maneira satisfatória de realização do trabalho, e não a melhor maneira de realizar o trabalho.

Na década de 60, a Teoria de Sistemas, conforme citado por Kurgant (1991), foi introduzida na análise organizacional, fundamentando-se na premissa de que os sistemas existem dentro de sistemas, são abertos e suas funções dependem de sua estrutura. Neste contexto, sistema é entendido como um conjunto de partes que se relacionam, cujos objetivos fazem com que o arranjo das partes não ocorra ao acaso. $\mathrm{O}$ homem se caracteriza pelo relacionamento interpessoal com outros indivíduos, e todos, na organização, são atores que representam papéis.

Em décadas mais recentes, a Teoria Contingencial surge como resposta a estudos que buscam entender como uma empresa se comporta em diferentes cenários, partindo do princípio de que a organização e o processo de trabalho de uma empresa são influenciados pelo ambiente externo em que se inserem.

Todas estas correntes do pensamento administrativo deixaram sua contribuição na organização das empresas modernas. Inicialmente, mediante a incorporação da racionalidade nas empresas e, gradativamente, da adaptação ao contexto social e da evolução e incorporação tecnológica. Em algumas mais, em outras menos, mas traços podem ser percebidos na estrutura das instituições que nos remetem à forma complexa de organização, tais como os vários níveis hierárquicos, concentração de poder e autonomia para tomada de decisão restrita aos que ocupam os cargos de chefia e direção. Mais recentemente, podem ser citadas a valorização do contexto social na definição do negócio da empresa, a flexibilização nos processos gerenciais, a mudança no processo de trabalho a partir da incorporação tecnológica e a participação do cliente/consumidor na definição do produto final.

Os modelos contemporâneos de administração, conduzidos por estratégias que permitem a flexibilização nos processos de produção, propiciam uma análise e um diagnóstico do ambiente, dando aos gerentes condições para antecipar o futuro e reduzir riscos e incertezas na tomada de decisão. As empresas deverão ser capazes de atender às demandas do mercado em tempo hábil, respondendo à clientela e ao avanço tecnológico, tornando suas empresas cada vez mais competitivas e garantindo, desta forma, o desenvolvimento institucional.

\section{MODELOS INOVADORES DE GERÊNCIA EM INSTITUIÇÕES DE SAÚDE}

$\mathrm{Na}$ área da saúde, algumas tentativas têm sido implementadas no sentido de buscar modelos de gerência alternativos aos modelos tradicionais de administração, em que a qualidade dos serviços prestados, a manutenção da organização e a satisfação dos trabalhadores devem ser garantidas.

As instituições prestadoras de serviços de saúde, em especial os hospitais, foram tradicionalmente organizadas à luz das teorias administrativas clássicas, o que pode ser constatado nas estruturas extremamente rígidas, com 
vários níveis hierárquicos, centralização do poder e limitada autonomia e responsabilidade dos níveis intermediários/gerenciais e operacionais, além da acentuada valorização da função controle, enquanto "fiscalização da produção".

A exemplo desta situação, citamos a lógica do desenho organizacional que mantém a dicotomia entre os diversos segmentos profissionais envolvidos com a atenção de saúde, com a criação de diretorias, departamentos, divisões, seções, setores, e que é reproduzida no interior de cada um destes níveis. Esta lógica privilegia o trabalho mecanizado, repetitivo, e cerceia a iniciativa e criatividade dos profissionais. A rigidez das normas e padrões define o que e como fazer o trabalho. Campos (1998), citando esta lógica tradicional de gerência, salienta ainda a elaboração centralizada de programas e normas reguladoras do atendimento e a quase ausência de comunicação horizontal e vertical dentro da organização.

Como conseqüência, o sistema de saúde atual está fragmentado no sentido da complementaridade da atenção à saúde, é caracterizado pelo alto consumo de recursos e baixa produtividade e não consegue responder às expectativas da população e dos profissionais envolvidos.

A atual proposição política para a área da saúde - Sistema Único de Saúde (SUS) - vislumbra uma reorganização técnica e gerencial dos serviços, por meio da adoção de modelos gerenciais flexíveis e a garantia da participação popular como estratégia de ação. No entanto, para que ocorram mudanças no sistema de saúde, é necessário haver uma transformação na prática das ações de saúde, por meio do envolvimento dos profissionais com o serviço e com o paciente, proporcionando maior resolutividade. Em conseqüência, espera-se uma transformação na forma de organização da instituição, para responder às necessidades do novo modelo gerencial.

Os novos modelos de gerência, de acordo com Campos (1998), sugerem modificações na estrutura da organização, extinguindo a departamentalização e criando unidades de produção conforme a lógica de cada processo de trabalho. Desta forma, todos os profissionais envolvidos com um determinado produto constituirão uma unidade de produção, ou seja, as equipes serão multiprofissionais.

No nosso entender, esta nova lógica de organização rompe com a dicotomia estabelecida na atenção ao paciente, em que "cada profissional faz a sua parte", possibilitando uma visão integral da assistência e a participação de todos os profissionais no novo desenho organizacional.
Neste novo desenho organizacional, a comunicação exerce papel fundamental para a articulação entre as unidades de produção, sendo o fluxo de comunicação lateral privilegiado em relação aos demais sentidos, uma vez que possibilita maior aproximação e articulação entre os serviços.

Diante destas transformações, é necessário que aconteçam também mudanças na forma de atuação dos gerentes, entendidos aqui como aqueles que respondem pela consecução dos objetivos da organização. Portanto, o gerente deve estar consciente da necessidade de redefinição do seu papel na organização.

\section{SISTEMAS DE INFORMACÃO PARA APOIO À DECISÃO NO EXERCÍCIO DA GERÊNCIA}

No processo de trabalho, a tomada de decisão é considerada a função que caracteriza o desempenho da gerência. Independentemente do aspecto da decisão, esta atitude deve ser fruto de um processo sistematizado, que envolve o estudo do problema a partir de um levantamento de dados, produção de informação, estabelecimento de propostas de soluções, escolha da decisão, viabilização e implementação da decisão e análise dos resultados obtidos.

No contexto organizacional, Choo (1998) considera que a tomada de decisão formal é estruturada por regras e procedimentos que especificam papéis, métodos e normas que, por sua vez, estabelecem valores que influenciam como a organização enfrenta a escolha e a incerteza. A combinação esperada entre cultura, comunicação e consenso melhora a eficiência e ajuda a alcançar um nível mais elevado de comportamento de escolha racional.

Nos vários modelos de decisão estudados, é possível reconhecer que a decisão nem sempre é resultado de um processo seqüencial, estruturado e dirigido para uma única solução. Mas é possível afirmar que a informação é um recurso primordial para a tomada de decisão e que, quanto mais estruturado for este processo, como no caso dos modelos racional e de processo, mais indicado se faz o uso de sistemas de informação que possam responder às demandas e necessidades informacionais do decisor. Da mesma forma, as informações requeridas para este tipo de decisão são mais objetivas e quantificáveis, tornando mais indicada a utilização de recursos informacionais que possam organizar, recuperar e disponibilizar as informações coletadas durante o processo de trabalho. Para os modelos de decisão em que a solução é resultado de um processo 
mais qualitativo, influenciado pelo "olhar" do decisor e por situações contingenciais, os sistemas de informação podem contribuir com dados que serão analisados e modificados para utilização na tomada de decisão.

O estudo da estrutura da organização permite conhecer o processo de comunicação formal e informal, reconhecendo-o como meio pelo qual os indivíduos se relacionam dentro da organização e como é empregado para apoiar as decisões, visando ao alcance dos objetivos institucionais. Nestes ambientes, valores são agregados à informação, transformando-a em matéria-prima para o desenvolvimento do produto da instituição. Seu objetivo principal é a busca da tomada de decisão certa, no momento oportuno, com as pessoas apropriadas, a partir da informação adequada, com o menor custo possível.

No contexto da tomada de decisão e considerando seu valor, a informação tem sido empregada como mais um recurso para o desenvolvimento do processo de trabalho nas organizações. A produção interna da informação e a utilização de fontes externas à organização suscitam a criação de sistemas de informação para sua identificação e organização, propiciando condições mais adequadas para sua recuperação e utilização na tomada de decisão.

No exercício da função gerencial, a ênfase deve ser dada à informação. Algumas estratégias são citadas por Davenport (1998) para o gerenciamento do comportamento informacional nos ambientes empresariais, dentre as quais se destacam tornar claros os objetivos e estratégias da organização, identificar competências informacionais, concentrar-se na administração de tipos específicos de conteúdos da informação, atribuir responsabilidades pelo comportamento informacional, criar uma rede de trabalho responsável pelo comportamento informacional e apresentar a todos os problemas do gerenciamento da informação.

Diante destas estratégias, é possível constatar que a informação é mais um recurso para a gerência nos ambientes empresariais e que é de todos os atores envolvidos no processo de trabalho a responsabilidade pela sua coleta, organização, distribuição e disponibilização. Desta forma, um sistema de informação que sirva ao processo de trabalho deve responder às demandas e necessidades dos diversos serviços e unidades da instituição, resguardadas suas características e especificidades, podendo ser únicos para a organização ou específicos para cada serviço.

Para efeito deste estudo, entende-se por sistema de informação todo conjunto de dados e informações que são organizados de forma integrada, com o objetivo de atender à demanda e antecipar as necessidades dos usuários. Portanto, sistemas de informação para apoio à decisão são sistemas que coletam, organizam, distribuem e disponibilizam a informação utilizada nesse processo.

Em geral, os sistemas de apoio à decisão obtêm dados do ambiente interno e externo à organização e processam estes dados, transformando-os em informações. O sistema opera por meio de softwares que permitem a disponibilização destas informações na forma de relatórios, de modelos matemáticos expressos em gráficos e tabelas e, ainda, permite que se instale um encontro virtual entre vários indivíduos trabalhando como um grupo dentro da organização. Além disso, como cita Richieri (2001), os softwares mais modernos permitem também a integração dos dados com resultados que refletem em maior rapidez na análise dos dados, transformando-os em informações essenciais para a tomada de decisão.

Os sistemas de informação nos ambientes empresariais são constituídos do gerenciamento da informação, a partir do levantamento das necessidades informacionais dos decisores, da coleta e obtenção dos dados, na análise dos dados transformando-os em informação, na distribuição da informação de acordo com as necessidades do decisor, da utilização das informações pela sua incorporação no processo de trabalho e, finalmente, da avaliação constante dos resultados obtidos e de redirecionamentos no sistema para atender às demandas e antecipar as necessidades dos decisores. É importante ressaltar que esses sistemas têm contribuído para o desenvolvimento do processo de produção nas instituições e que, nos ambientes hospitalares, em especial, têm possibilitado maior segurança para a tomada de decisão, o que resulta em melhor atendimento aos pacientes.

Diante do exposto, o objetivo desta pesquisa é analisar a utilização de sistemas de informação, enquanto instrumento para a tomada de decisão no exercício da gerência de unidades funcionais do Hospital das Clínicas da Universidade Federal de Minas Gerais.

\section{METODOLOGIA}

Esta investigação foi desenvolvida por meio de um estudo qualitativo e descritivo, caracterizando, no primeiro momento, a gerência, nas unidades de produção, a tomada de decisão no exercício da gerência e realizando um levantamento e análise das informações utilizadas pelo gerente na tomada de decisão. Com estes dados e já no segundo momento, descrevemos o ambiente informacional do gerente, bem como apontamos diretrizes para criação 
e a automação de sistemas de informação enquanto instrumento da gerência.

O campo de estudo escolhido foi o Hospital das Clínicas da Universidade Federal de Minas Gerais (HC/UFMG). Para realização da pesquisa foi concedida a aprovação do projeto no âmbito institucional, junto à Diretoria de Ensino, Pesquisa e Extensão do HC/UFMG e Comitê de Ética em Pesquisa da UFMG.

Para este estudo, foram selecionadas as unidades funcionais de caráter assistencial que já assinaram ou se organizaram para firmar o contrato de gestão e, entre estas, aquelas que mantêm semelhança na estrutura organizacional, no que diz respeito às coordenações de enfermagem, médica e administrativa e, no processo assistencial, quanto à média de permanência dos pacientes e demanda e complexidade do cuidado requerido - cuidado intermediário.

Os sujeitos foram os profissionais que desenvolvem ações de gerência da unidade. Obtivemos a colaboração de 19 profissionais, sendo sete enfermeiros, quatro médicos, um gerente de unidade funcional e sete coordenadores administrativos. A pesquisa foi realizada em dois momentos distintos.

O primeiro momento constituiu-se de observação do cotidiano dos serviços na unidade funcional, com ênfase na estrutura gerencial da unidade, buscando caracterizar a função dos gerentes na unidade por meio das ações que desenvolvem e que os distinguem dos demais profissionais da unidade. Com esta estratégia, buscamos presenciar o processo de trabalho na unidade e estabelecer um contato com os profissionais, por meio de idas consecutivas ao campo de estudo, antes da fase intensiva de coleta de dados realizada por entrevista.

Na etapa de observação, foi utilizado um diário de campo para registro das observações, considerando a data da observação, a unidade observada, o período de observação e os comentários e impressões da pesquisadora. O segundo momento constituiu-se da entrevista semiestruturada, buscando traçar um perfil dos sujeitos da pesquisa por meio do levantamento de dados de identificação, tais como idade, profissão, tempo de formado, titulação profissional, tempo de trabalho na instituição e tempo de exercício na função de gerente. A segunda parte da entrevista foi composta de perguntas norteadoras que se referiam diretamente às informações produzidas pelos coordenadores, necessidades de informação para o exercício da gerência, fontes de informação utilizadas e fontes potenciais de informação. A organização dos dados foi realizada com base na análise temática de conteúdo proposta por Bardin (1977), que consta de três fases distintas: pré-análise; exploração do material; tratamento dos resultados, a inferência e a interpretação.

\section{APRESENTAC̣ÃO E DISCUSSÃO DOS RESULTADOS}

No período de observação das atividades desempenhadas pelos gerentes e coordenadores, percebemos que não foram significativas as alterações no processo de gerência da unidade com base no modelo de unidades funcionais. Decidimos, então, iniciar a realização das entrevistas, conforme cronograma elaborado, considerando que a implementação do novo modelo de gerência era ainda mais incipiente nas demais unidades.

$\mathrm{Na}$ caracterização das atividades desempenhadas pelos gerentes, foi possível verificar que a gerência nem sempre é (re)conhecida como uma função exclusiva do profissional que ocupa o cargo de gerente, mas, sim, como uma atividade desenvolvida por aqueles que coordenam o grupo profissional a que pertencem. $\mathrm{Ou}$ seja, a gerência da unidade funcional ainda mantém vestígios do modelo de coordenação de enfermagem, médica e administrativa. Em algumas unidades, é possível perceber experiências de um trabalho conjunto e participativo e, em outras, uma ação dirigida apenas aos profissionais da área específica.

As ações apontadas como características da função de coordenação referem-se aos recursos tradicionais da administração - materiais, humanos, físicos, financeiros e de comunicação - e são voltadas, na sua maioria, para as ações burocráticas, tendo maior destaque as atividades administrativas propriamente ditas, que envolvem a organização, coordenação e direção das ações desempenhadas na unidade, com ênfase na gerência de recursos humanos.

Embora implantado na instituição desde 1999, este modelo ainda não gerou mudanças significativas no processo de trabalho nas unidades estudadas. Em algumas delas, percebemos iniciativas de uma organização colegiada como diretriz para o processo de trabalho; em outras, apenas a nomeação de um gerente que ainda não exerce plenamente sua função e, em outras, pelo momento incipiente de transformação, não foi possível perceber mudanças no processo de trabalho.

Os coordenadores e gerentes diferenciam sua função dos demais profissionais da unidade, pela responsabilidade em tomar decisões no âmbito da unidade funcional. Esta ação é resultante de um processo sistematizado de 
identificação do problema, levantamento de possibilidades de solução e implementação de medidas que visam à solução do problema. Na prática, vários são os fatores intervenientes que influenciam no alcance das soluções almejadas, e, entre estes, foram identificados pelos gerentes e coordenadores a estrutura organizacional, as relações estabelecidas entre os diferentes serviços, o sistema de comunicação formal e informal, assim como alguns instrumentos de gerência.

Este estudo permitiu identificar que, em especial, os coordenadores de enfermagem demonstraram maior conhecimento e domínio das condições de trabalho e de assistência na unidade. Este fato é decisivo para a mudança proposta, pois a escolha do gerente não pode, de antemão, limitar-se à formação acadêmica nem à representatividade social e institucional que possui o profissional. É necessário que o gerente tenha um domínio do processo de trabalho e seja capaz de estabelecer relações significativas na organização, que concorram para uma tomada de decisão mais acertada e, conseqüentemente, para melhor resultado do trabalho. Para tanto, faz-se necessário desenvolver a habilidade gerencial e a capacidade de utilizar instrumentos de todos os profissionais, de modo que este aprimoramento facilite e apóie a gerência.

O sistema de informação é apontado como um destes instrumentos, embora não se encontre organizado a ponto de responder às demandas e necessidades de informação para a tomada de decisão pelos gerentes. Para os gerentes e coordenadores, a informação é a base para a tomada de decisão, e, para que a solução sugerida aos problemas seja efetiva e acertada, as informações devem ser precisas, relevantes, oportunas, permitindo uma abordagem completa da situação.

Os dados e informações comumente utilizados são aqueles que se referem ao cuidado do paciente e aos recursos organizacionais disponíveis para a coordenação da unidade, em especial, os recursos humanos. A compreensão do significado de necessidades e fontes de informação reafirma que a maior dificuldade para criação de um sistema de informação está na definição das necessidades e demandas por parte dos usuários, pois, por várias vezes, foram citadas fontes como necessidades de informação para a tomada de decisão.

Tendo como referência o cenário vivido pelos gerentes e coordenadores na unidade funcional e o ambiente informacional disponível, ousa-se pontuar alguns subsídios para a (re)organização da informação como recurso para a gerência, considerando os usuários do sistema, as demandas e necessidades de informação, as fontes disponíveis e potenciais.

O ponto de partida é o repensar da prática gerencial, ou seja, as mudanças geradas no processo de trabalho pela incorporação tecnológica, que vão requerer também mudanças na forma como se faz o trabalho. Em especial quando se opta por utilizar sistemas informatizados, isto não significa que o modo como são organizados os dados e as informações produzidas no processo de trabalho não seja transferido para o meio eletrônico.

O desenvolvimento de sistemas de informação prevê a abordagem das normas relativas aos aspectos estruturais da organização e da especificidade técnica de cada serviço. No que se refere à terminologia, deve-se adotar um sistema de classificação que permita a organização, o acesso e a recuperação das informações para utilização no processo de trabalho. Alguns aspectos devem ser observados: definir regras para as classificações, de forma a assegurar a uniformidade no seu emprego; incorporar expressões pertinentes à determinada categoria; assegurar que as categorias dos termos sejam excludentes; garantir a estabilidade do sistema para possibilitar uma análise comparativa ao longo do tempo.

Com base nos resultados deste estudo, pode-se constatar que a tomada de decisão é pautada tanto em dados assistenciais quanto em dados organizacionais. Considerando a natureza das informações produzidas na assistência ao paciente, há indícios de que as classificações existentes não alcançam representar, em nível adequado, todos os tipos de dados que geralmente são frutos do processo assistencial. Em contrapartida, os dados referentes à organização são mais objetivos e, supostamente, de fácil organização.

É importante ressaltar que o processo de trabalho na instituição implica, necessariamente, a articulação entre os diversos setores. Portanto, não se trata de criar sistemas isolados para cada serviço, mas sim garantir a articulação dos dados, orientada pela especificidade de cada serviço e pela seletividade no acesso à informação. Acrescido a estas condições, o sistema deve utilizar componentes estratégicos que reúnam condições de aplicabilidade, sustentabilidade e orientação para os objetivos para o qual foi definido. 


\section{Eliane Marina Palhares Guimarães / Yolanda Dora Martinez Évora}

\section{CONCLUSÃO}

Sem a intenção de esgotar a discussão do tema, o estudo possibilitou repensar a função gerencial nas unidades funcionais, a partir de um modelo que privilegia a descentralização administrativa e técnica da unidade, a participação na tomada de decisão e o trabalho compartilhado entre os profissionais que ali atuam. $\mathrm{Na}$ busca de identificar instrumentos que pudessem auxiliar no exercício da gerência, o sistema de informação foi estudado considerando as informações produzidas, os usuários do sistema e suas demandas e necessidades, as fontes mais utilizadas e as potenciais.

O estudo permite concluir que o modelo gerencial de unidades funcionais está em diferentes fases de implementação, nas unidades funcionais estudadas, e que o sistema de informação disponível não está estruturado para responder às necessidades dos gerentes. Quanto à informatização, existem experiências de sistemas operando em serviços específicos, de forma desintegrada, e, neste momento, a instituição inicia a instalação de um sistema que busca responder às demandas dos gerentes e à integração das informações produzidas no âmbito organizacional.

Artigo recebido em 05-12-2003 e aceito para publicação em 24-04-2004.

\section{REFERENNCIAS}

BARDIN, L. Análise de conteúdo. Tradução de Luis Antero Reto e Augusto Pinheiro. Lisboa : Edições 70, 1977. 229 p.

CAMPOS, G. W. S. O anti-Taylor: sobre a invenção de um método para co-governar instituições de saúde produzindo liberdade e compromisso. Cadernos Saúde Pública, Rio de Janeiro, v. 14, n. 4, p. 863-870, out./dez. 1998.

$\mathrm{CHOO}, \mathrm{C} . \mathrm{W}$. The management of uncertainty: organizations as decision-making systems. In: _______. The knowing organizations: how organizations use information to construct meaning, create knowledge, and make decisions. New York : Oxford University, 1998. p. 155. 205.

DAVENPORT, T. Ecologia da informação: por que só a tecnologia não basta para o sucesso na era da informação. São Paulo : Futura, 1998. $316 \mathrm{p}$.

MOTTA, F. C. P. Teoria das organizações: evolução e crítica. São Paulo : Pioneira, 1986. $112 \mathrm{p}$

MOTTA, P. R. Transformação organizacional: a teoria e a prática de inovar. Rio de Janeiro : Qualitymark, 1999a. 224 p.

OLIVEIRA, M. de Energia emocional. São Paulo : Makron Books, 1997. 220 p.

KURGANT, P. As teorias de administração e os serviços de enfermagem. In: EPU, 1991. cap. 1, p. 3-13. Administração em enfermagem. São Paulo :

RICHIERI, F. L. A nova geração de ferramentas de TI. Gazeta Mercantil, São Paulo, 22 fev. 2001 


\section{ANEXO \\ SISTEMAS DE INFORMAÇÃO: INSTRUMENTO PARA TOMADA DE DECISÃO NO EXERCÍCIO DA GERÊNCIA}

Pesquisadora: Profa. Eliane Marina Palhares Guimarães

\section{DIÁRIO DE CAMPO}

Data: 1

Unidade:

Período de observação:

Observações e impressões da pesquisadora:

\section{ROTEIRO PARA ENTREVISTA}

Título da Pesquisa: Sistemas de informação: instrumento para tomada de decisão no exercício da gerência

1. Dados pessoais:

1.1. Sexo:
( ) Feminino
( ) Masculino

1.2. Faixa etária:
( ) até 30 anos
( ) de 31 a 40 anos
( ) de 41 a 50 anos
( ) mais de 50 anos 
2. Dados profissionais:

2.1. Profissão:

2.2. Titulação:

2.2.1. ( ) graduação

2.2.2. ( ) especialização

2.2.3. ( ) mestrado

2.2.4. ( ) doutorado

2.3. Cargo ocupado na instituição:

2.4. Horário de trabalho:

2.5. Tempo de trabalho na instituição:

2.5.1. ( ) de 1 a 5 anos

2.5.2. ( ) de 6 a 10 anos

2.5.3. ( ) de 11 a 15 anos

2.5.4. ( ) mais de 15 anos

2.6. Tempo que está no cargo de gerência:

\section{Questões norteadoras:}

3.1. Quais as atividades que caracterizam a função de gerência na unidade funcional?

3.2. No exercício da gerência, quais as atividades que podem ser consideradas como uma ação de tomada de decisão?

3.3. Quais as informações que você gera como gerente de unidade?

3.4. Quem utiliza as informações geradas por você?

3.5. Quais as informações necessárias para a tomada de decisão?

3.6. Quais as fontes de informação disponíveis para obtenção das informações necessárias?

3.7. As fontes de informação disponíveis atendem às necessidades de informação?

3.8. É possível identificar fontes de informação em potencial? Quais? 